\title{
SYNCHRONIZATION POINTS AND ASSOCIATED DYNAMICAL INVARIANTS
}

\author{
RICHARD MILES
}

\begin{abstract}
This paper introduces new invariants for multiparameter dynamical systems. This is done by counting the number of points whose orbits intersect at time $n$ under simultaneous iteration of finitely many endomorphisms. We call these points synchronization points. The resulting sequences of counts together with generating functions and growth rates are subsequently investigated for homeomorphisms of compact metric spaces, toral automorphisms and compact abelian group epimorphisms. Synchronization points are also used to generate invariant measures and the distribution properties of these are analysed for the algebraic systems considered. Furthermore, these systems reveal strong connections between the new invariants and problems of active interest in number theory, relating to heights and greatest common divisors.
\end{abstract}

\section{INTRODUCTION}

Invariants play a central role in dynamical systems and ergodic theory, one of the best known being entropy. For a hyperbolic toral automorphism $\alpha$, the topological (equivalently, metric) entropy $h(\alpha)$ is related to the growth rate of periodic points by the formula

$$
\lim _{n \rightarrow \infty}\left|F_{n}(\alpha)\right|^{1 / n}=\exp (h(\alpha)),
$$

where $F_{n}(\alpha)$ is the set of points fixed by the map $\alpha^{n}$ and $|\cdot|$ denotes cardinality (see 32 for details). This result extends in a subtle way to many other nonhyperbolic epimorphisms of compact abelian groups [8] and to an extensively studied class of multiparameter dynamical systems of algebraic origin [17. Nonetheless, many of the most natural multiparameter examples, such as algebraic $\mathbb{Z}^{d}$-actions on tori and solenoids, $d \geqslant 2$, have zero entropy and it is natural to explore alternative, more revealing invariants in such cases.

To this end, invariants such as directional entropy 27, 5, 10, Friedland entropy [14, 15], [10], the Lind dynamical zeta function [19] and the orbit growth function [26] have been applied with varying levels of success. For example, a convenient formula exists for directional entropy for the examples described above [10, Sec. 8], whereas the orbit growth function behaves erratically and proves difficult to work with [26, Sec. 3]. From an alternative perspective, originating from the concepts of expansive subdynamics and entropy rank [5], [11, a good level of understanding now exists concerning the relationship between periodic orbits of individual maps in a $\mathbb{Z}^{d}$-action and the action itself (see [22], 24], [25] for instance).

Received by the editors March 13, 2011 and, in revised form, March 4, 2012.

2010 Mathematics Subject Classification. Primary 37A35, 37B05, 37C25, 37C85, 37P30, 11G50, 11Z05. 
In light of the disparity between these perspectives, and with broader applicability in mind, this paper explores the middle ground by introducing a new set of invariants based not on periodic orbits of individual maps but upon a more general concept of synchronization, definable for any finite set of endomorphisms $\Lambda$, $|\Lambda| \geqslant 2$, of a common phase space $X$. First, consider two endomorphisms $\alpha, \beta$ of $X$, iterated to produce two discrete time dynamical systems. An aspect of shared behaviour of these maps is encoded by counting the number of points $x \in X$ that have synchronous orbits at time $n \in \mathbb{N}$, in the sense that

$$
\alpha^{n}(x)=\beta^{n}(x) \text {. }
$$

More generally, for a finite set of endomorphisms $\Lambda$ on $X$ with $|\Lambda| \geqslant 2$, define

$$
S_{n}(\Lambda)=\left\{x \in X: \alpha^{n}(x)=\beta^{n}(x) \text { for some } \alpha, \beta \in \Lambda, \alpha \neq \beta\right\}
$$

and

$$
S_{n}^{\star}(\Lambda)=\left\{x \in X: \alpha^{n}(x)=\beta^{n}(x) \text { for all } \alpha, \beta \in \Lambda, \alpha \neq \beta\right\} .
$$

These two sets will be termed the weak synchronization points and strong synchronization points for $\Lambda$ at time $n$, respectively. They are readily verified to be finite sets for many familiar dynamical systems.

If $\Lambda$ contains the identity map, $S_{n}^{\star}(\Lambda)$ consists of the points simultaneously fixed by the $n$-th iterates of the non-trivial maps in $\Lambda$, so $S_{n}^{\star}(\alpha, \mathrm{Id})=S_{n}(\alpha, \mathrm{Id})=F_{n}(\alpha)$ and hence synchronization points generalize periodic points. We will be particularly interested in the integer sequences $\left(\left|S_{n}(\Lambda)\right|\right)$ and $\left(\left|S_{n}^{\star}(\Lambda)\right|\right)$, the existence and value of the growth rates

$$
r_{\Lambda}=\lim _{n \rightarrow \infty}\left|S_{n}(\Lambda)\right|^{1 / n}, \quad r_{\Lambda}^{\star}=\lim _{n \rightarrow \infty}\left|S_{n}^{\star}(\Lambda)\right|^{1 / n}
$$

and the ordinary generating functions

$$
\sigma_{\Lambda}(z)=\sum_{n=1}^{\infty}\left|S_{n}(\Lambda)\right| z^{n}, \quad \sigma_{\Lambda}^{\star}(z)=\sum_{n=1}^{\infty}\left|S_{n}^{\star}(\Lambda)\right| z^{n} .
$$

Notice that if $r_{\Lambda}$ exists, then $r_{\Lambda}^{-1}$ is the radius of convergence of $\sigma_{\Lambda}$ (similarly for $r_{\Lambda}^{\star}$ and $\left.\sigma_{\Lambda}^{\star}\right)$.

This paper is organized as follows. Section 2 introduces some fundamental properties, examples and general results. Using results of Bowen 4, for certain commuting homeomorphisms of a compact metric space, a formula for $r_{\Lambda}$ is obtained relating to topological entropy (Theorem 2.2). The prototypical case of automorphisms of the two torus is considered in Section 3 . There, formulae for $\left|S_{n}(\alpha, \beta)\right|$ are found without assuming that $\alpha$ and $\beta$ commute, and $\sigma_{\alpha, \beta}$ is seen to be a rational function in all reasonable cases (Theorem 3.1). Theorem 3.1 is then applied to obtain $r_{\Lambda}$ for $|\Lambda| \geqslant 3$ (Corollary 3.2). In Section 4, our attention is turned to commuting compact abelian group epimorphisms. In this case, bounds for $\left|S_{n}(\Lambda)\right|$ reveal a connection between $r_{\Lambda}$ and projective heights (Theorem 4.7). The results also show that invertibility plays a central role in this respect (see Remarks 4.8). Strong synchronization is also considered and, for the class of $S$-integer dynamical systems [8, a formula is obtained for $\left|S_{n}^{\star}(\Lambda)\right|$ (Theorem 4.1) that leads to a circle of problems of active interest in number theory; this becomes the focus in Section 5

By applying a powerful result of Corvaja and Zannier [9], it is shown that $|\Lambda| \geqslant 3$ implies $r_{\Lambda}^{\star}=1$ in many cases considered in Section 4 (Theorem 5.2). In terms of weak synchronization, their bound also allows us to show that $\sigma_{\Lambda}$ has meromorphic continuation to the open unit disc for commuting toral automorphisms, provided 
the associated $\langle\Lambda\rangle$-action is mixing (Theorem [5.3). To conclude, motivated by the results of Bowen [3, invariant measures related to synchronization are considered and well-known theorems concerning uniform distribution of periodic point measures [18, 8] are extended to synchronization point measures (Theorem [5.5). Indeed, our results expand what is known for periodic point measures. Finally, synchronization point measures lead us to consider the consequences of a conjecture of Ailon and Rudnick [2].

\section{Fundamental properties, examples and general Results}

In this section, some fundamental properties of $S_{n}(\Lambda)$ and $S_{n}^{\star}(\Lambda)$ are assembled. Synchronization is considered for some well-known examples and, in particular, for a set $\Lambda$ of commuting homeomorphisms of a compact metric space, a formula is given relating the growth rate $r_{\Lambda}$ to topological entropy. Throughout, it is assumed that $\Lambda$ is a set containing at least two maps.

Let $X, Y$ be sets and $\Lambda_{X}, \Lambda_{Y}$ finite sets of endomorphisms of $X$ and $Y$, respectively, in one-to-one correspondence, $\alpha_{X} \leftrightarrow \alpha_{Y}, \alpha_{X} \in \Lambda_{X}, \alpha_{Y} \in \Lambda_{Y}$. Suppose that $\phi: X \rightarrow Y$ is a bijection such that $\phi \alpha_{X}(x)=\alpha_{Y} \phi(x)$ for all $x \in X$ and all correspondents $\alpha_{X}, \alpha_{Y}$. Then, for any $n \in \mathbb{N}$, and distinct $\alpha_{X}, \beta_{X} \in \Lambda_{X}$,

$$
\alpha_{X}^{n}(x)=\beta_{X}^{n}(x) \Leftrightarrow \alpha_{Y}^{n} \phi(x)=\beta_{Y}^{n} \phi(x) .
$$

Hence, $S_{n}\left(\Lambda_{Y}\right)=\phi\left(S_{n}\left(\Lambda_{X}\right)\right)$ and $S_{n}^{\star}\left(\Lambda_{Y}\right)=\phi\left(S_{n}^{\star}\left(\Lambda_{X}\right)\right)$. This means that $r_{\Lambda}, r_{\Lambda}^{\star}$, $\sigma_{\Lambda}$ and $\sigma_{\Lambda}^{\star}$ are invariants of bijective conjugacy.

Proposition 2.1. Let $\Lambda$ be a finite set of endomorphisms of a set $X, n \in \mathbb{N}$ and let $\mathcal{M}$ be the collection of all 2-element subsets of $\Lambda$. Assume that for any distinct $\alpha, \beta \in \Lambda, S_{n}(\alpha, \beta)$ is finite.

(1) If $|\Lambda|=2, S_{n}(\Lambda)=S_{n}^{\star}(\Lambda)$.

(2) For any distinct $\alpha, \beta \in \Lambda,\left|S_{n}^{\star}(\Lambda)\right| \leqslant\left|S_{n}(\alpha, \beta)\right|$.

(3) For any distinct $\alpha, \beta \in \Lambda,\left|S_{n}(\Lambda)\right| \geqslant\left|S_{n}(\alpha, \beta)\right|$.

(4)

$$
\left|S_{n}(\Lambda)\right|=\sum_{\mathcal{L} \subset \mathcal{M}}(-1)^{|\mathcal{L}|-1}\left|I_{n}(\mathcal{L})\right|,
$$

(5)

where, for $\mathcal{L} \subset \mathcal{M}, I_{n}(\mathcal{L})=\bigcap_{\{\alpha, \beta\} \in \mathcal{L}} S_{n}(\alpha, \beta)$.

$$
\max _{\{\alpha, \beta\} \in \mathcal{M}}\left|S_{n}(\alpha, \beta)\right| \leqslant\left|S_{n}(\Lambda)\right| \leqslant 2^{|\mathcal{M}|} \max _{\{\alpha, \beta\} \in \mathcal{M}}\left|S_{n}(\alpha, \beta)\right| .
$$

Proof. (11)-(3) follow immediately from the definitions.

(4) Upon noticing that

$$
S_{n}(\Lambda)=\bigcup_{\{\alpha, \beta\} \in \mathcal{M}} S_{n}(\alpha, \beta),
$$

the result follows from the inclusion-exclusion principle.

(5) The first inequality follows from (31). For the second inequality, since for any $\mathcal{L} \subset \mathcal{M}$ we have

$$
\left|I_{n}(\mathcal{L})\right| \leqslant \max _{\{\alpha, \beta\} \in \mathcal{L}}\left|S_{n}(\alpha, \beta)\right| \leqslant \max _{\{\alpha, \beta\} \in \mathcal{M}}\left|S_{n}(\alpha, \beta)\right|,
$$

the result follows from (4). 
Now suppose that $\Lambda$ is a set of commuting automorphisms of a set $X$, so that given any distinct $\alpha, \beta \in \Lambda$, for all $x \in X$,

$$
\alpha^{n}(x)=\beta^{n}(x) \Leftrightarrow\left(\beta^{-1} \alpha\right)^{n}(x)=x .
$$

Therefore,

$$
S_{n}(\alpha, \beta)=F_{n}\left(\beta^{-1} \alpha\right) .
$$

This observation can be readily applied in the case of expansive homeomorphisms on a compact metric space $(X, d)$. Recall that a homeomorphism $\alpha$ of $X$ is expansive if there exists a constant $\varepsilon>0$ such that given any two distinct points $x, y \in X$, there exists $n \in \mathbb{Z}$ such that $d\left(\alpha^{n}(x), \alpha^{n}(y)\right)>\varepsilon$.

Theorem 2.2. Let $\Lambda$ be a finite set of commuting homeomorphisms of a compact metric space $X$ such that for any two distinct maps $\alpha, \beta \in \Lambda$, the homeomorphism $\beta^{-1} \alpha$ is expansive. Then

$$
r_{\Lambda}=\max _{\alpha, \beta \in \Lambda, \alpha \neq \beta}\left\{\exp \left(h\left(\beta^{-1} \alpha\right)\right)\right\},
$$

where $h$ denotes the topological entropy.

Proof. Let $\Lambda^{\prime}=\left\{\beta^{-1} \alpha: \alpha, \beta \in \Lambda\right.$ and $\left.\alpha \neq \beta\right\}$. For any $\gamma \in \Lambda^{\prime}$, by [4, Lem. 4], there exist positive constants $A_{\gamma}, B_{\gamma}$ such that, for sufficiently large $n$,

$$
A_{\gamma} \exp (h(\gamma) n) \leqslant\left|F_{n}(\gamma)\right| \leqslant B_{\gamma} \exp (h(\gamma) n) .
$$

Furthermore, by Proposition 2.1(5) and (2.1), there is a positive constant $C$ such that

Therefore, for sufficiently large $n$,

$$
\max _{\gamma \in \Lambda^{\prime}}\left|F_{n}(\gamma)\right| \leqslant\left|S_{n}(\Lambda)\right| \leqslant C \max _{\gamma \in \Lambda^{\prime}}\left|F_{n}(\gamma)\right| .
$$

$$
A_{\Lambda}^{1 / n} \exp (\lambda) \leqslant\left|S_{n}(\Lambda)\right|^{1 / n} \leqslant B_{\Lambda}^{1 / n} \exp (\lambda),
$$

where $A_{\Lambda}=\max \left\{A_{\gamma}: \gamma \in \Lambda^{\prime}\right\}, B_{\Lambda}=C \max \left\{B_{\gamma}: \gamma \in \Lambda^{\prime}\right\}$ and $\lambda=\max \{h(\gamma): \gamma \in$ $\left.\Lambda^{\prime}\right\}$.

The assumption that the maps in $\Lambda$ commute endows sets of synchronization points with $\Lambda$-invariance. In particular, if $x \in S_{n}(\Lambda)$, then for some distinct $\alpha, \beta \in$ $\Lambda, \alpha^{n}(x)=\beta^{n}(x)$. Hence, for any $\gamma \in \Lambda$,

$$
\alpha^{n}(\gamma(x))=\gamma \alpha^{n}(x)=\gamma \beta^{n}(x)=\beta^{n}(\gamma(x)),
$$

which implies $\gamma(x) \in S_{n}(\Lambda)$, and so $S_{n}(\Lambda)$ is $\Lambda$-invariant. When $S_{n}(\Lambda)$ is finite, it must therefore be comprised of pre-periodic and periodic points of any given map in $\Lambda$. Similar statements are true for $S_{n}^{\star}(\Lambda)$.

If it is further assumed that the maps in $\Lambda$ are invertible, then one can easily construct $\Lambda$-invariant measures for finite $S_{n}^{\star}(\Lambda)$ in the following way:

$$
\nu_{\Lambda, n}^{\star}=\left|S_{n}^{\star}(\Lambda)\right|^{-1} \sum_{x \in S_{n}^{\star}(\Lambda)} \delta_{x},
$$

where $\delta_{x}$ is the Dirac mass at $x$. The distribution properties of these synchronization point measures 1 may be studied in a similar way to those of periodic point measures, and we will do this for commuting compact group automorphisms in Section 5 .

Our attention is now turned to some familiar examples.

\footnotetext{
${ }^{1}$ One may also define the measure $\nu_{\Lambda, n}$ using the weak synchronization points in an analogous way, but we do not consider this here.
} 
Example 2.3 (Two epimorphisms of the torus $\mathbb{T}$ and invertible extensions). Consider the epimorphisms $x \mapsto 2 x, x \mapsto 3 x$ of the torus $X=\mathbb{T}$. The synchronization points for $\Lambda=\{\times 2, \times 3\}$ at time $n \in \mathbb{N}$ are the solutions to the equation $2^{n} x=3^{n} x$ mod 1 . Hence, we obtain a Lucas sequence,

$$
\left|S_{n}(\Lambda)\right|=3^{n}-2^{n},
$$

$r_{\Lambda}=\lim _{n \rightarrow \infty}\left(3^{n}-2^{n}\right)^{1 / n}=3$ and

$$
\sigma_{\Lambda}(z)=\frac{1}{1-3 z}-\frac{1}{1-2 z} .
$$

The natural extension on which both $\times 2$ and $\times 3$ are invertible is the Pontryagin dual of the ring $\mathbb{Z}\left[\frac{1}{6}\right]$, which is locally isomorphic to $\mathbb{R} \times \mathbb{Q}_{2} \times \mathbb{Q}_{3}$. On this space, (2.1) together with [23, Th. 1.1] show that again

$$
\left|S_{n}(\Lambda)\right|=\left|F_{n}\left(\times \frac{2}{3}\right)\right|=3^{n}-2^{n} .
$$

Some care is required when passing to invertible extensions. Consider the pair of epimorphisms $\Lambda=\{\times 3, \times 6\}$ on $X=\mathbb{T}$. Then,

$$
\left|S_{n}(\Lambda)\right|=6^{n}-3^{n}, \sigma_{\Lambda}(z)=\frac{1}{1-6 z}-\frac{1}{1-3 z} \text { and } r_{\Lambda}=6 .
$$

However, when $\left.X=\widehat{\mathbb{Z}\left[\frac{1}{6}\right.}\right]$, (2.1) and [23, Th. 1.1] show that

$$
\left|S_{n}(\Lambda)\right|=\left|F_{n}(\times 2)\right|=\left(2^{n}-1\right)\left|2^{n}-1\right|_{3},
$$

where $|\cdot|_{3}$ is the 3 -adic valuation. Therefore, $r_{\Lambda}=2$. Furthermore, the sequence $\left(\left|S_{n}(\Lambda)\right|\right)$ cannot be a recurrence sequence (see for example, [8, Sec. 8]), and so $\sigma_{\Lambda}$ is irrational. For further details about this sequence in a dynamical context, see [12.

Example 2.4 (Three epimorphisms of the torus $\mathbb{T}$ ). Now suppose $X=\mathbb{T}$ and $\Lambda=\{\times a, \times b, \times c\}$, where $a<b<c$ are positive integers. Then Proposition 2.1(4) shows that

$$
\begin{aligned}
\left|S_{n}(a, b, c)\right| & =\left|S_{n}(a, b)\right|+\left|S_{n}(a, c)\right|+\left|S_{n}(b, c)\right|-2\left|S_{n}^{\star}(a, b, c)\right| \\
& =b^{n}-a^{n}+c^{n}-a^{n}+c^{n}-b^{n}-2\left|S_{n}^{\star}(a, b, c)\right| \\
& =2\left(c^{n}-a^{n}-\left|S_{n}^{\star}(a, b, c)\right|\right),
\end{aligned}
$$

and since $\left|S_{n}^{\star}(a, b, c)\right| \leqslant\left|S_{n}(a, b)\right| \leqslant b^{n}$, this gives $r_{\Lambda}=c$. Furthermore, a straightforward duality argument (see Lemma 4.2) shows that

$$
\left|S_{n}^{\star}(a, b, c)\right|=\left|\mathbb{Z} /\left(c^{n}-a^{n}, b^{n}-a^{n}\right)\right|=\operatorname{gcd}\left(c^{n}-a^{n}, b^{n}-a^{n}\right) .
$$

Sequences of this nature feature prominently in a circle of problems in number theory. For example, it is well known that $\left|S_{n}^{\star}(1,2,3)\right|$ is unbounded, but it is not known whether or not $\left|S_{n}^{\star}(1,2,3)\right|=1$ infinitely often (see Conjecture 5.7). A simple experiment with a computer program will reveal the erratic nature of this sequence. Nonetheless, Bugeaud, Corvaja and Zannier [6] show that given any $\varepsilon>0$, there are only finitely many exceptions to the inequality

$$
\left|S_{n}^{\star}(1,2,3)\right|=\operatorname{gcd}\left(3^{n}-1,2^{n}-1\right)<\exp (\varepsilon n) .
$$

A consequence of this bound is that $r_{1,2,3}^{\star}=1$. Moreover, in Section 5 it will be shown that the unit circle is a natural boundary for $\sigma_{1,2,3}^{\star}$. Using (2.3),

$$
\sigma_{1,2,3}(z)=\frac{4 z}{(1-3 z)(1-z)}-2 \sigma_{1,2,3}^{\star}(z)
$$


TABLE 1. Synchronization point counts for pairs of automorphisms of $\mathbb{T}^{2}$.

$$
\begin{array}{c|c|c}
A & B & \left|S_{n}(A, B)\right| \\
\left.\hline \begin{array}{ll}
2 & 1 \\
1 & 0
\end{array}\right) & \left(\begin{array}{ll}
3 & 2 \\
1 & 1
\end{array}\right) & \frac{5-2 \sqrt{2} \sqrt{3}}{4 \sqrt{2} \sqrt{3}}((1+\sqrt{2})(2+\sqrt{3}))^{n}+\sum_{\omega} c_{\omega} \omega^{n} \\
\left(\begin{array}{ll}
2 & 1 \\
1 & 0
\end{array}\right) & \left(\begin{array}{ll}
-3 & -2 \\
8 & 5
\end{array}\right) & \left(\frac{\sqrt{2}}{2} n+1\right)(1+\sqrt{2})^{n}-\left(\frac{\sqrt{2}}{2} n-1\right)(1-\sqrt{2})^{n} \\
\left(\begin{array}{ll}
2 & -1 \\
9 & -4
\end{array}\right) & \left(\begin{array}{ll}
-3 & -2 \\
8 & 5
\end{array}\right) & 50 n^{2}-2+2(-1)^{n} \\
\left(\begin{array}{ll}
2 & 1 \\
-7 & -3
\end{array}\right) & \left(\begin{array}{cc}
-3 & -2 \\
8 & 5
\end{array}\right) & \begin{cases}\beth_{1} \\
2 n+\left(\xi^{-1}-\xi\right)\left(\xi^{n}-\xi^{-n}\right) & 3 \nmid n\end{cases}
\end{array}
$$

which is meromorphic inside the unit circle.

Example 2.5 (A full-shift and a flip). One of the simplest examples of two noncommuting maps on the same space which generate interesting dynamics is that of a full-shift and a flip (see for example [16]). Let $\mathcal{A}$ be a finite alphabet, $A=|\mathcal{A}|$ and $X=\mathcal{A}^{\mathbb{Z}}$. For $x=\left(x_{j}\right) \in X$, the shift map $\alpha: X \rightarrow X$ and the flip $\beta: X \rightarrow X$ are defined by

$$
\alpha(x)_{j}=x_{j+1} \text { and } \beta(x)_{j}=x_{-j} .
$$

A straightforward calculation gives

$$
\left|S_{n}(\alpha, \beta)\right|= \begin{cases}A^{n}, & n \text { even } \\ A^{(n+1) / 2}, & n \text { odd }\end{cases}
$$

Therefore,

$$
\begin{aligned}
\sigma_{\alpha, \beta}(z) & =\sum_{n=1}^{\infty}\left|S_{2 n}(\alpha, \beta)\right| z^{2 n}+\sum_{n=1}^{\infty}\left|S_{2 n-1}(\alpha, \beta)\right| z^{2 n-1} \\
& =\sum_{n=1}^{\infty}(A z)^{2 n}+z^{-1} \sum_{n=1}^{\infty}\left(A z^{2}\right)^{n} \\
& =\frac{A^{2} z^{2}}{(1-A z)(1+A z)}+\frac{A z}{\left(1-A^{1 / 2} z\right)\left(1+A^{1 / 2} z\right)}
\end{aligned}
$$

Example 2.6 (Automorphisms of the two torus $\mathbb{T}^{2}$ ). Automorphisms of $\mathbb{T}^{2}$ are given by $2 \times 2$ invertible matrices with integer entries and determinant \pm 1 . Let $A$ and $B$ be two such matrices (not necessarily assumed to commute). In the next section, this situation will be considered more generally and it will be shown that, provided $A^{n}-B^{n}$ is non-singular,

$$
\left|S_{n}(A, B)\right|=\left|\operatorname{ker}\left(A^{n}-B^{n}\right)\right|=\left|\operatorname{det}\left(A^{n}-B^{n}\right)\right| .
$$

This quantity has been calculated for four pairs of matrices in Table 1 . In the first example, the dominant term in the sequence is shown and $\omega$ runs through the remaining products from the set

$$
\left\{\omega_{1} \omega_{2}:\left\{\omega_{1}, \omega_{2}\right\} \subset\{1+\sqrt{2}, 1-\sqrt{2}, 2+\sqrt{3}, 2-\sqrt{3}\}\right\} .
$$


Note that $B$ is an isometry in the second example and both $A$ and $B$ are isometries in the third and fourth examples. In the fourth example, $A^{3}=\mathrm{Id}$, and since $B^{n} x=x$ for all points of the form $x=(t,-2 t) \in \mathbb{T}^{2}, S_{n}(A, B)$ is uncountably infinite whenever $3 \mid n$. When $3 \nmid n$, the formula given expresses $\left|S_{n}(A, B)\right|$ in terms of $\xi=\exp (2 \pi i / 3)$. In all the other examples, $S_{n}(A, B)$ is finite for all $n \in \mathbb{N}$ and the associated generating function $\sigma_{A, B}$ may be calculated using the formula given for $\left|S_{n}(A, B)\right|$. Therefore, it is a rational function in each case. In the first example, there is one simple pole on the circle of convergence $|z|=(-1+\sqrt{2})(2-\sqrt{3})$, one double pole on $|z|=-1+\sqrt{2}$ in the second example and one triple pole on $|z|=1$ in the third.

To conclude this section, we consider an example where the number of strong synchronization points grows more rapidly than in any of the examples thus far. In particular, $\sigma_{\Lambda}^{\star}$ has radius of convergence zero.

Example 2.7 (Shift extensions of $\times 2, \times 3$ ). Let $X=\mathbb{T}^{\mathbb{N}}$ and define endomorphisms $\alpha, \beta, \gamma$ of $X$ by

$$
\alpha(x)_{j}=2 x_{j}, \beta(x)_{j}=3 x_{j} \text { and } \gamma(x)_{j}=x_{j+1},
$$

where $x=\left(x_{j}\right) \in X$. Let $S_{n}(2,3)$ denote the synchronization points at time $n$ for $\times 2, \times 3$ on $\mathbb{T}$. Then

$$
S_{n}^{\star}(\alpha, \beta, \gamma)=\left\{x=\left(x_{j}\right) \in S_{n}(2,3)^{\mathbb{N}}: x_{j+n}=x_{j}\right\} .
$$

Since $\left|S_{n}(2,3)\right|=3^{n}-2^{n}$, it follows that $\left|S^{\star}(\alpha, \beta, \gamma)\right|=\left(3^{n}-2^{n}\right)^{n}$, and so

$$
\left|S^{\star}(\alpha, \beta, \gamma)\right|^{1 / n}=3^{n}-2^{n} \rightarrow \infty .
$$

\section{Automorphisms of the two torus}

The purpose of the theorem below is to describe the variety of possible growth rates of synchronization points in a familiar setting, allowing the possibility of non-commuting maps. For ease, we consider two matrices $A, B \in \mathrm{SL}(2, \mathbb{Z})$ as no new phenomena are witnessed by allowing negative determinants. Denote the number of synchronization points of the corresponding automorphisms of $X=\mathbb{T}^{2}$ by $S_{n}(A, B)$. Let $\lambda, \delta$ and $\mu, \varepsilon$ be the respective pairs of eigenvalues of $A$ and $B$ and, where possible, choose $\lambda$ and $\mu$ to denote the eigenvalues of maximum modulus in each pair. Set $s_{A}=\operatorname{sgn}(\operatorname{tr}(A))$ and $s_{B}=\operatorname{sgn}(\operatorname{tr}(B))$. Note that the assumption $A^{n}-B^{n}$ is non-singular is needed to ensure that $S_{n}(A, B)$ is finite.

Theorem 3.1. For all $n \in \mathbb{N}$ such that $A^{n}-B^{n}$ is non-singular,

(1) If $A$ and $B$ are both hyperbolic,

$$
\left|S_{n}(A, B)\right|=\left|\sum_{\omega} c_{\omega} \omega^{n}\right|,
$$

where $\omega$ runs through the set $\left\{\omega_{1} \omega_{2}:\left\{\omega_{1}, \omega_{2}\right\} \subset\{\lambda, \delta, \mu, \varepsilon\}\right\}$ and $c_{\omega} \in$ $\mathbb{Q}(\lambda, \mu)$. Moreover, if $\operatorname{deg}(\mathbb{Q}(\lambda, \mu) \mid \mathbb{Q})=4$, then $c_{\lambda \mu} \neq 0$ and

$$
\left|S_{n}(A, B)\right|=\left|c_{\lambda \mu}\right||\lambda \mu|^{n}+\mathrm{O}\left(\max \{|\lambda|,|\mu|\}^{n}\right) .
$$

(2) If $A$ is hyperbolic and $B$ is not hyperbolic,

$$
\left|S_{n}(A, B)\right|=\psi(n) \lambda^{n}+\mathrm{O}(n),
$$


where $\psi$ is a non-trivial linear polynomial if $B$ is parabolic and non-trivial or where $\psi$ is non-trivial and periodic otherwise.

(3) If both $A$ and $B$ are parabolic and non-trivial,

$$
\left|S_{n}(A, B)\right|=\left|\operatorname{det}\left(A-s_{A} s_{B} B\right)\right| n^{2}+\mathrm{O}(1) .
$$

(4) If $A$ is parabolic and non-trivial and $B$ has finite order, then

$$
\left|S_{n}(A, B)\right|=\psi(n) n+\mathrm{O}(1),
$$

where $\psi(n)=\left|\operatorname{tr}\left(B^{n}\right)-s_{A} \operatorname{tr}\left(A B^{-n}\right)\right|$, which is a periodic function.

Furthermore, if $A^{n}-B^{n}$ is non-singular for all $n \in \mathbb{N}$, then $\sigma_{A, B}$ is rational.

Proof. If $A^{n}-B^{n}$ is non-singular, then

$$
\begin{aligned}
\left|S_{n}(A, B)\right| & =\left|\operatorname{ker}\left(A^{n}-B^{n}\right)\right| \\
& =\left|\operatorname{ker}\left(B^{-n} A^{n}-\mathrm{Id}\right)\right| \\
& =\left|\operatorname{det}\left(B^{-n} A^{n}-\mathrm{Id}\right)\right| \\
& =\left|\operatorname{det}\left(A^{n}-B^{n}\right)\right|,
\end{aligned}
$$

where the third equality follows from the standard fixed point formula for a toral automorphism.

(1) Suppose that $P$ diagonalizes $A$ and $Q$ diagonalizes $B$, so that

$$
\operatorname{det}\left(A^{n}-B^{n}\right)=\operatorname{det}\left(P\left(\begin{array}{ll}
\lambda^{n} & 0 \\
0 & \delta^{n}
\end{array}\right) P^{-1}-Q\left(\begin{array}{ll}
\mu^{n} & 0 \\
0 & \varepsilon^{n}
\end{array}\right) Q^{-1}\right) .
$$

Equation (3.1) is obtained by evaluating this determinant (which is a lengthy but straightforward calculation).

Since $|\operatorname{det}(A)|=|\operatorname{det}(B)|=1,|\lambda|,|\mu|>1$ and $0<|\delta|,|\varepsilon|<1$. Therefore, to obtain (3.2), it is sufficient to show that $c_{\lambda \mu} \neq 0$.

Write $A=\left(a_{i j}\right)$ and $B=\left(b_{i j}\right)$ and note that $P$ takes one of the forms

$$
\left(\begin{array}{ll}
\lambda-a_{22} & \delta-a_{22} \\
a_{21} & a_{21}
\end{array}\right) \text { if } a_{21} \neq 0, \quad\left(\begin{array}{ll}
a_{12} & a_{12} \\
\lambda-a_{11} & \delta-a_{11}
\end{array}\right) \text { if } a_{21}=0
$$

observing that $a_{12} \neq 0$ in the latter case. The entries of $Q$ are determined analogously, depending on whether or not $b_{21}=0$. Calculating (3.3) gives

$$
c_{\lambda \mu}=\frac{\eta(A, B)}{(\mu-\epsilon)(\delta-\lambda) a_{21} b_{21}}
$$

where

$$
\eta(A, B)=\left\{\begin{array}{l}
\left(b_{21}\left(\delta-a_{22}\right)-a_{21}\left(\varepsilon-b_{22}\right)\right)\left(b_{21}\left(\lambda-a_{22}\right)-a_{21}\left(\mu-b_{22}\right)\right), \\
\left(a_{12} b_{21}-\left(\delta-a_{11}\right)\left(\varepsilon-b_{22}\right)\right)\left(\left(\lambda-a_{11}\right)\left(\mu-b_{22}\right)-a_{12} b_{21}\right), \\
\left(b_{12}\left(\delta-a_{11}\right)-a_{12}\left(\varepsilon-b_{11}\right)\right)\left(b_{12}\left(\lambda-a_{11}\right)-a_{21}\left(\mu-b_{11}\right)\right),
\end{array}\right.
$$

depending on the three cases: $a_{21} \neq 0, b_{21} \neq 0$ or $a_{21}=0, b_{21} \neq 0$ or $a_{21}=0, b_{21}=$ 0 , respectively. In the first case, if $c_{\lambda \mu}=0$, then

$$
b_{21}\left(\delta-a_{22}\right)=a_{21}\left(\varepsilon-b_{22}\right) \text { or } b_{21}\left(\lambda-a_{22}\right)=a_{21}\left(\mu-b_{22}\right) .
$$

However, both these equalities are precluded since $\mu, \epsilon \notin \mathbb{Q}(\lambda)=\mathbb{Q}(\delta)$, giving a contradiction. Therefore, $c_{\lambda \mu} \neq 0$. The other two cases are dealt with similarly.

(2) Suppose that $P$ diagonalizes $A$. This time, consider

$$
\operatorname{det}\left(A^{n}-B^{n}\right)=\operatorname{det}\left(P\left(\begin{array}{ll}
\lambda^{n} & 0 \\
0 & \delta^{n}
\end{array}\right) P^{-1}-B^{n}\right) .
$$


If $B$ is parabolic (so, $\operatorname{tr}(B)= \pm 2$ ) and $B \neq \pm \mathrm{Id}$, we have

$$
B^{n}=s_{B}^{n}\left(\left(s_{B} B-\mathrm{Id}\right) n+\mathrm{Id}\right) .
$$

Calculating the right hand side of (3.5) gives

$$
\operatorname{det}\left(A^{n}-B^{n}\right)=\left(c n+s_{B} \frac{a(\lambda-\delta)}{\operatorname{det}(P)}\right) \lambda^{n}+\psi_{1}(n) \delta^{n}+\psi_{2}(n),
$$

where $c$ is a rational constant, $\psi_{1}$ and $\psi_{2}$ are linear polynomials and $a=a_{12}$ or $a=a_{21}$, depending on the form of $P$ (see (3.4) $)$. Note that $a(\lambda-\delta) \neq 0$.

The remaining case is that $B= \pm \operatorname{Id}$ or $B$ is elliptic, so $B^{n}=\operatorname{Id}$ for some $n \in \mathbb{N}$. In this case, the right hand side of (3.5) is

$$
\frac{1}{\operatorname{det}(P)}\left(\psi_{1}(n) \lambda^{n}+\psi_{2}(n) \delta^{n}+2\right),
$$

where $\psi_{1}$ and $\psi_{2}$ are periodic functions whose periods are at most the order of $B$, and $\psi_{1}(n)=-\operatorname{det}(P) \neq 0$ when $B^{n}=\mathrm{Id}$.

(3) In this case, both $\operatorname{tr}(A)= \pm 2$ and $\operatorname{tr}(B)= \pm 2$. If $s_{A}=s_{B}$, then

$$
\begin{aligned}
\operatorname{det}\left(A^{n}-B^{n}\right) & =\operatorname{det}\left(s_{A}^{n}\left(\left(s_{A} A-\mathrm{Id}\right) n+\mathrm{Id}\right)-s_{B}^{n}\left(\left(s_{B} B-\mathrm{Id}\right) n+\mathrm{Id}\right)\right) \\
& =\operatorname{det}(A-B) n^{2} .
\end{aligned}
$$

If $s_{A} \neq s_{B}$, without loss of generality, assume $s_{A}=1$ and $s_{B}=-1$, so

$$
\begin{aligned}
\operatorname{det}\left(A^{n}-B^{n}\right) & =\operatorname{det}\left(n(A-\mathrm{Id})+\mathrm{Id}-(-1)^{n+1}(n(B+\mathrm{Id})-\mathrm{Id})\right) \\
& = \begin{cases}\operatorname{det}(A-B-2(1-1 / n) \mathrm{Id}) n^{2}, & n \text { odd }, \\
\operatorname{det}(A+B) n^{2}, & n \text { even. }\end{cases}
\end{aligned}
$$

Since

$$
\begin{aligned}
& \operatorname{det}(A+B)+\operatorname{det}(A-B-2(1-1 / n) \mathrm{Id}) \\
= & 2(\operatorname{det}(A)+\operatorname{det}(B))-2(1-1 / n)(\operatorname{tr}(A)-\operatorname{tr}(B)-2(1-1 / n)) \\
= & \frac{4}{n^{2}},
\end{aligned}
$$

it follows that

$$
\operatorname{det}\left(A^{n}-B^{n}\right)= \begin{cases}4-\operatorname{det}(A+B) n^{2}, & n \text { odd } \\ \operatorname{det}(A+B) n^{2}, & n \text { even }\end{cases}
$$

which gives the required estimate for $\left|S_{n}(A, B)\right|$.

(44) In the final case, since $\operatorname{tr}(A)= \pm 2$,

$$
\begin{aligned}
\operatorname{det}\left(A^{n}-B^{n}\right) & =\operatorname{det}\left(s_{A}^{n}\left(\left(s_{A} A-\mathrm{Id}\right) n+\mathrm{Id}\right)-B^{n}\right) \\
& =s_{A}^{n}\left(\operatorname{tr}\left(B^{n}\right)-s_{A} \operatorname{tr}\left(A B^{-n}\right)\right) n-s_{A}^{n} \operatorname{tr}\left(B^{n}\right)+2,
\end{aligned}
$$

again giving the required estimate for $\left|S_{n}(A, B)\right|$.

Finally, in each of the cases above, note that the exact expressions obtained for $\operatorname{det}\left(A^{n}-B^{n}\right)$ show that $\sigma_{A, B}$ is rational, provided $A^{n}-B^{n}$ is non-singular for all $n \in \mathbb{N}$.

In many situations, the theorem above combined with Proposition 2.1(5) allows one to deduce exact asymptotics for $\left|S_{n}(\Lambda)\right|$ (and therefore the value of $r_{\Lambda}$ ) when $|\Lambda| \geqslant 3$. The dominant term will simply be the dominant synchronization point count $\left|S_{n}(A, B)\right|$, as $A$ and $B$ run through distinct pairs of matrices in $\Lambda$. This will 
occur for a fixed pair for all sufficiently large $n$, provided the maps in $\Lambda$ are sufficiently distinctive, for example, if the number fields generated by the eigenvalues of each automorphism are distinct. Hence, we have the following.

Corollary 3.2. Let $\Lambda$ be a finite set of automorphisms of $\mathbb{T}^{2}$ and suppose that the number fields generated by the eigenvalues of each automorphism are distinct. Then

$$
r_{\Lambda}=\max _{\alpha, \beta \in \Lambda, \alpha \neq \beta}\{\exp (h(\alpha)+h(\beta))\} .
$$

In general, estimates for $\left|S_{n}^{\star}(\Lambda)\right|$ are considerably harder to obtain when $|\Lambda| \geqslant 3$. This problem is considered for commuting automorphisms in the next section.

Finally, the following example shows that (3.2) does not necessarily hold if $\operatorname{deg}(\mathbb{Q}(\lambda, \mu) \mid \mathbb{Q})=2$.

Example 3.3. Let

$$
A=\left(\begin{array}{ll}
8 & 13 \\
13 & 21
\end{array}\right) \text { and } B=\left(\begin{array}{ll}
3 & 5 \\
5 & 8
\end{array}\right)
$$

The corresponding eigenvalues are

$$
\lambda=\frac{29}{2}+\frac{13}{2} \sqrt{5}, \delta=\frac{29}{2}-\frac{13}{2} \sqrt{5}, \mu=\frac{11}{2}+\frac{5}{2} \sqrt{5}, \varepsilon=\frac{11}{2}-\frac{5}{2} \sqrt{5},
$$

and

$$
\left|S_{n}(A, B)\right|=\left|\operatorname{det}\left(A^{n}-B^{n}\right)\right|=\left|-(\lambda \varepsilon)^{n}-(\mu \delta)^{n}+2(-1)^{n}\right| .
$$

So, in contrast to the case $\operatorname{deg}(\mathbb{Q}(\lambda, \mu) \mid \mathbb{Q})=4$, the growth in $\left|S_{n}(A, B)\right|$ is not dominated by the product of the two eigenvalues of maximum modulus. Instead,

$$
\left|S_{n}(A, B)\right|=|\lambda \varepsilon|^{n}+\mathrm{O}(1)
$$

\section{Counting SynChronization Points FOR COMMUTING GROUP EPIMORPHISMS}

For commuting epimorphisms of a compact abelian group, there is a sizeable tool box based on techniques from commutative algebra [30] and algebraic number theory, especially for counting problems (see for example 8 and 23]). Furthermore, the structure of systems in which each of the generators has finite positive entropy is particularly well understood [10], and this now motivates the focus on epimorphisms with finite entropy.

Throughout this section, $\Lambda$ is a finite set of commuting epimorphisms of a compact metrizable abelian group $X$. If $M=\widehat{X}$ is the Pontryagin dual of $X$, then $M$ becomes a module over the polynomial ring $R_{\Lambda}=\mathbb{Z}\left[t_{\alpha}: \alpha \in \Lambda\right]$ by identifying each dual automorphism $\widehat{\alpha}, \alpha \in \Lambda$ with multiplication by $t_{\alpha}$ and extending this in a natural way to polynomials. Conversely, by duality, any module over a polynomial ring in finitely many variables with integer coefficients gives rise to a finite set $\Lambda$ of commuting epimorphisms of a compact metrizable abelian group $X=\widehat{M}$. A full introduction to this framework in the case that each map is invertible is given in Schmidt's monograph [30]. However, invertibility is not necessary for many of the fundamental connections with commutative algebra developed there.

A global field is either a finite extension of $\mathbb{Q}$ or a finite extension of a function field of the form $\mathbb{F}_{p}(t)$. If $\mathfrak{p} \subset R_{\Lambda}$ is a prime ideal such that $R_{\Lambda} / \mathfrak{p}$ has krull dimension 1 (denoted $\operatorname{kdim}\left(R_{\Lambda} / \mathfrak{p}\right)=1$ ), the field of fractions of this domain is a 
global field $\mathbb{K}(\mathfrak{p})$. These domains are central to the study of finite entropy epimorphisms [10. The places $\mathcal{P}(\mathbb{K})$ of a global field $\mathbb{K}$ are the equivalence classes of absolute values on $\mathbb{K}$. When $\operatorname{char}(\mathbb{K})=0$, the infinite places are the archimedean ones. When $\operatorname{char}(\mathbb{K})>0$, all places are non-archimedean and the infinite places comprise the extensions of the infinite place of $\mathbb{F}_{p}(t)$, given by $|f / g|_{\infty}=p^{\operatorname{deg}(f)-\operatorname{deg}(g)}$. All other places are said to be finite. Given a finite place of $\mathbb{K}$, there corresponds a unique discrete valuation $v$ whose precise value group is $\mathbb{Z}$. The corresponding normalized absolute value is

$$
|\cdot|_{v}=\left|\mathfrak{K}_{v}\right|^{-v(\cdot)},
$$

where $\mathfrak{K}_{v}$ is the (necessarily finite) residue class field of $v$.

The following theorem gives a strong synchronization point counting formula for the $S$-integer dynamical systems of Chothi, Everest and Ward [8]. These systems encompass examples such as Examples 2.3 and 2.4 together with a rich supply of toral automorphisms, shift maps and various extensions.

Theorem 4.1. Let $\mathbb{K}$ be a global field, $S \subset \mathcal{P}(\mathbb{K})$ a set of finite places,

$$
D=\left\{x \in \mathbb{K}:|x|_{v} \leqslant 1 \text { for all finite } v \notin S\right\}
$$

(the ring of $S$-integers in $\mathbb{K}$ ) and $\Lambda$ a finite subset of non-zero elements of $D$ such that $\mu^{-1} \lambda$ is not a root of unity for all $\mu, \lambda \in \Lambda, \mu \neq \lambda$. If $S_{n}^{\star}(\Lambda)$ is the set of strong synchronization points for the maps dual to multiplication by $\lambda \in \Lambda$ on $D$, then

$$
\left|S_{n}^{\star}(\Lambda)\right|=\prod_{v} \min \left\{\left|\mu^{n}-\lambda^{n}\right|_{v}^{-1}: \lambda \in \Lambda, \lambda \neq \mu\right\},
$$

where $v$ runs through all finite $v \notin S$ and we may freely choose $\mu \in \Lambda$.

When $\Lambda$ is a finite set of epimorphisms of a compact abelian group $X$, it is straightforward to show that $S_{n}^{\star}(\Lambda)$ is a subgroup of $X$. Via Pontryagin duality, this allows one to express the cardinality of $S_{n}^{\star}(\Lambda)$ in terms of the dual module. Let $\mathfrak{a}_{n}=\mathfrak{a}_{n}(\Lambda)$ denote the ideal $\left(t_{\alpha}^{n}-t_{\beta}^{n}: \alpha, \beta \in \Lambda, \alpha \neq \beta\right) \subset R_{\Lambda}$.

Lemma 4.2. Let $\Lambda$ be a finite set of epimorphisms of a compact abelian group $X$ and let $M$ be the dual $R_{\Lambda}$-module. Then whenever either

$$
S_{n}^{\star}(\Lambda) \text { or } M / \mathfrak{a}_{n} M
$$

is finite, these groups have the same cardinality.

Proof. Since

$$
S_{n}^{\star}(\Lambda)=\bigcap_{\alpha, \beta \in \Lambda, \alpha \neq \beta} \operatorname{ker}\left(\alpha^{n}-\beta^{n}\right)
$$

and $\operatorname{ker}\left(\alpha^{n}-\beta^{n}\right)^{\perp}=\left(t_{\alpha}^{n}-t_{\beta}^{n}\right) M$, it follows that

$$
S_{n}^{\star}(\Lambda)^{\perp}=\sum_{\alpha, \beta \in \Lambda, \alpha \neq \beta}\left(t_{\alpha}^{n}-t_{\beta}^{n}\right) M=\mathfrak{a}_{n} M .
$$

The result follows because $\widehat{S_{n}^{\star}(\Lambda)} \cong M / S_{n}^{\star}(\Lambda)^{\perp}$ and $S_{n}^{\star}(\Lambda) \cong \widehat{S_{n}^{\star}(\Lambda)}$ whenever either of these groups is finite.

Proof of Theorem 4.1. Since $D$ is a ring of $S$-integers, note that any proper quotient ring of $D$ is finite. By Lemma 4.2, it suffices to calculate $\left|D / \overline{\mathfrak{a}}_{n}\right|$, where $\overline{\mathfrak{a}}_{n}$ is the 
image of $\mathfrak{a}_{n}$ in $D$. Note that

$$
\overline{\mathfrak{a}}_{n}=\left(\mu^{n}-\lambda^{n}: \mu, \lambda \in \Lambda, \lambda \neq \mu\right)=\left(\mu^{n}-\lambda^{n}: \lambda \in \Lambda, \lambda \neq \mu\right)
$$

for any fixed choice of $\mu \in \Lambda$ and that $\overline{\mathfrak{a}}_{n} \neq\{0\}$ as $\mu^{n} \neq \lambda^{n}$ for all $\lambda \in \Lambda$.

By finding a composition series for $D / \overline{\mathfrak{a}}_{n}$ as a $D$-module and successively localizing at each of its associated primes to obtain multiplicities, we find

$$
\left|D / \overline{\mathfrak{a}}_{n}\right|=\prod_{\mathfrak{m} \in \operatorname{Ass}\left(D / \overline{\mathfrak{a}}_{n}\right)}|D / \mathfrak{m}|^{d(\mathfrak{m}, \Lambda)},
$$

where

$$
d(\mathfrak{m}, \Lambda)=\operatorname{dim}_{D / \mathfrak{m}}\left(D / \overline{\mathfrak{a}}_{n}\right)_{\mathfrak{m}} .
$$

Since $D$ is a ring of $S$-integers, $\operatorname{kdim}(D)=1$ and [21, Th 12.5] and [21, Th. 6.5] show that the set of prime ideals $\mathfrak{m} \subset D$ for which $\left(D / \overline{\mathfrak{a}}_{n}\right)_{\mathfrak{m}} \neq\{0\}$ is precisely $\operatorname{Ass}\left(D / \overline{\mathfrak{a}}_{n}\right)$. Therefore, the product in (4.2) may be taken over all non-trivial prime ideals $\mathfrak{m} \subset D$ to yield the same result. Furthermore, $D_{\mathfrak{m}}$ is a discrete valuation ring and hence a principal ideal domain, so $\left(\overline{\mathfrak{a}}_{n}\right)_{\mathfrak{m}}$ collapses to a principal ideal in $D_{\mathfrak{m}}$ and

$$
\begin{aligned}
d(\mathfrak{m}, \Lambda) & =\operatorname{dim}_{D / \mathfrak{m}}\left(D / \overline{\mathfrak{a}}_{n}\right)_{\mathfrak{m}} \\
& =\operatorname{dim}_{D / \mathfrak{m}}\left(D_{\mathfrak{m}} /\left(\overline{\mathfrak{a}}_{n}\right)_{\mathfrak{m}}\right) \\
& =\min \left\{v_{\mathfrak{m}}\left(\mu^{n}-\lambda^{n}\right): \lambda \in \Lambda, \lambda \neq \mu\right\}
\end{aligned}
$$

where $v_{\mathfrak{m}}$ denotes the normalized valuation corresponding to $\mathfrak{m}$. Upon noting that

$$
|\cdot|_{\mathfrak{m}}^{-1}=|D / \mathfrak{m}|^{v_{\mathfrak{m}}(\cdot)}
$$

the required formula for $\left|D / \overline{\mathfrak{a}}_{n}\right|$ follows from the modified form of the product in (4.2) which runs over all non-trivial prime ideals $\mathfrak{m} \subset D$, as these are in one-toone correspondence with the finite places not included in $S$.

Example 4.3 (Example 3.3 revisited). Since the automorphisms in Example 3.3 commute, it can easily be reformulated in terms of Theorem 4.1. Let

$$
D=\left\{x \in \mathbb{Q}(\sqrt{5}):|x|_{v} \leqslant 1 \text { for all finite } v \in \mathcal{P}(\mathbb{Q}(\sqrt{5}))\right\}=\mathbb{Z}\left[\frac{1}{2}(1+\sqrt{5})\right],
$$

and let $\lambda, \delta, \mu, \varepsilon$ be as in Example 3.3 . The map $\phi: D \rightarrow \mathbb{Z}^{2}$, given by

$$
\phi(a+b(1+\sqrt{5}) / 2)=(a, b),
$$

is an isomorphism whose dual simultaneously conjugates the maps dual to multiplication by $\lambda$ and $\mu$ to multiplication by the matrices $A$ and $B$ on $\mathbb{T}^{2}$ given in Example 3.3. Since there are only two maps to consider, Theorem 4.1 and the product formula for global fields give

$$
\left|S^{\star}(\lambda, \mu)\right|=\prod_{v<\infty}\left|\mu^{n}-\lambda^{n}\right|_{v}^{-1}=\left|\mu^{n}-\lambda^{n}\right|_{\infty}\left|\tau(\mu)^{n}-\tau(\lambda)^{n}\right|_{\infty},
$$

where $\tau$ is the non-trivial element of $\operatorname{Gal}(\mathbb{Q}(\sqrt{5}) \mid \mathbb{Q})$ and $|\cdot|_{\infty}$ is the usual real absolute value. Since $\tau(\mu)=\varepsilon$ and $\tau(\lambda)=\delta$,

$$
\left|S^{\star}(\lambda, \mu)\right|=\left|(\lambda \delta)^{n}-(\lambda \varepsilon)^{n}-(\mu \delta)^{n}+(\mu \varepsilon)^{n}\right|_{\infty}=\left|-(\lambda \varepsilon)^{n}-(\mu \delta)^{n}+2(-1)^{n}\right|,
$$

as before. 
When considering just two epimorphisms $\alpha$ and $\beta$, calculation of the cardinality of modules of the form $M / \mathfrak{a}_{n} M$ is aided by the principality of $\mathfrak{a}_{n}=\left(t_{\alpha}^{n}-t_{\beta}^{n}\right)$. In fact, it is fairly straightforward to modify the proof of the periodic point counting formula [23, Th. 1.1] to obtain a formula for $\left|S_{n}(\alpha, \beta)\right|$ in the generality assumed there. However, for ease, assume that $X$ is connected and finite dimensional in what follows.

Method 4.4 (Canonical filtration). Let $\Lambda$ be a finite set of epimorphisms of $X$ and let $M$ be the dual $R_{\Lambda}$-module. For any associated prime $\mathfrak{p} \in \operatorname{Ass}(M)$, let $\lambda_{\alpha}(\mathfrak{p})$ denote the image of $t_{\alpha}$ in $R_{\Lambda} / \mathfrak{p}$. Since $X$ is assumed to be connected, this means $M$ is torsion-free as a group and $\operatorname{kdim}\left(R_{\Lambda} / \mathfrak{p}\right)>0$ for all $\mathfrak{p} \in \operatorname{Ass}(M)$, as otherwise there is an embedding of a finite field $R_{\Lambda} / \mathfrak{p}$ into $M$. In addition, since $X$ is finite dimensional, the set of associated primes $\operatorname{Ass}(M)$ is finite and comprises primes $\mathfrak{p} \subset R_{\Lambda}$ such that $\mathfrak{p} \cap \mathbb{Z}=\{0\}$ and $\operatorname{kdim}\left(R_{\Lambda} / \mathfrak{p}\right)=1$, so the field of fractions of $R_{\Lambda} / \mathfrak{p}$ is an algebraic number field.

Let $U=\mathbb{Z} \backslash\{0\}$. Localizing at $U$, the natural map $M \rightarrow U^{-1} M$ is injective (since $M$ is a torsion-free group) and $U^{-1} M$ is a Noetherian module over $Q_{\Lambda}=$ $\mathbb{Q}\left[t_{\alpha}: \alpha \in \Lambda\right]$ (since finite dimension of $X$ implies $M$ has finite rational rank). Furthermore, there is a prime filtration

$$
\{0\}=M_{0} \subset M_{1} \subset \cdots \subset M_{r}=U^{-1} M,
$$

in which $M_{i} / M_{i-1} \cong Q_{\Lambda} / \mathfrak{q}_{i}$ for a list of maximal ideals $\mathfrak{q}_{1}, \ldots, \mathfrak{q}_{r} \subset Q_{\Lambda}$ with

$$
\left\{\mathfrak{q}_{i} \cap R_{\Lambda}: 1 \leqslant i \leqslant r\right\}=\operatorname{Ass}(M) .
$$

Identifying $M$ with its canonical image in $U^{-1} M$ and intersecting the chain (4.3) with $M$ gives a chain

$$
\{0\}=L_{0} \subset L_{1} \subset \cdots \subset L_{r}=M .
$$

Considering (4.4) as a chain of $R_{\Lambda}$-modules, for each $1 \leqslant i \leqslant r$ there is an induced inclusion

$$
\frac{R_{\Lambda}}{\mathfrak{q}_{i} \cap R_{\Lambda}} \hookrightarrow \frac{L_{i}}{L_{i-1}} \hookrightarrow \frac{M_{i}}{M_{i-1}} \cong \frac{Q_{\Lambda}}{\mathfrak{q}_{i}},
$$

by which the quotient $L_{i} / L_{i-1}$ naturally becomes a module of the domain on the left and embeds in its field of fractions, $\mathbb{K}_{i}$ say, which identifies with the field on the right. Set

$$
P_{i}=\left\{v \in \mathcal{P}\left(\mathbb{K}_{i}\right):|\cdot|_{v} \text { is bounded on } L_{i} / L_{i-1}\right\}
$$

and

$$
\lambda_{\alpha, i}=\lambda_{\alpha}\left(\mathfrak{q}_{i} \cap R_{\Lambda}\right) .
$$

Note that every $v \in P_{i}$ is finite.

The canonical filtration described above, together with the subsequent sets of places, will be used to analyse the growth rate of weak synchronization points.

Lemma 4.5. Suppose that for all distinct $\alpha, \beta \in \Lambda$ and all $1 \leqslant i \leqslant r, \lambda_{\alpha, i}^{n} \neq \lambda_{\beta, i}^{n}$. Then

$$
\left|S_{n}(\alpha, \beta)\right|=\prod_{i=1}^{r} \prod_{v \in P_{i}}\left|\lambda_{\alpha, i}^{n}-\lambda_{\beta, i}^{n}\right|_{v}^{-1}
$$


Furthermore, for any collection $\mathcal{L}$ of 2-element subsets of $\Lambda$,

$$
\left|I_{n}(\mathcal{L})\right| \leqslant C \prod_{i=1}^{r} \prod_{v \in P_{i}} \min \left\{\left|\lambda_{\alpha, i}^{n}-\lambda_{\beta, i}^{n}\right|_{v}^{-1}:\{\alpha, \beta\} \in \mathcal{L}\right\},
$$

where $I_{n}(\mathcal{L})$ is as in Proposition 2.1 and $C$ is a positive constant depending only on $M$.

Remark 4.6. The condition that $\lambda_{\alpha, i}^{n} \neq \lambda_{\beta, i}^{n}$ for all $1 \leqslant i \leqslant r$ ensures that $\left|S_{n}(\alpha, \beta)\right|$ is finite for all $n \in \mathbb{N}$. This will be the case if, for example, the semi-group action generated by $\Lambda$ is mixing (see [30, Th. 6.5]). However, it is a weaker condition. For example, the lemma applies when $X=\mathbb{T}$ and $\Lambda=\{\times 2, \times 4\}$, giving $\left|S_{n}(2,4)\right|=$ $2^{n}\left(2^{n}-1\right)$, but the action generated by $\Lambda$ is not mixing. If $\alpha$ and $\beta$ are invertible, the condition is equivalent to ergodicity of $\beta^{-1} \alpha$.

Proof of Lemma 4.5. To obtain the formula for $\left|S_{n}(\alpha, \beta)\right|=\left|M /\left(t_{\alpha}^{n}-t_{\beta}^{n}\right) M\right|$, proceed (carefully) as in [23, Sec. 4] with the polynomial $t_{\alpha}^{n}-t_{\beta}^{n}$ in place of $t_{\alpha}^{n}-1$ that is used there.

To obtain the upper bound for $\left|I_{n}(\mathcal{L})\right|$, as in Lemma 4.2, first observe that $\left|I_{n}(\mathcal{L})\right|=\left|M / \mathfrak{a}_{n} M\right|$, where $\mathfrak{a}_{n}$ is the ideal $\left(t_{\alpha}^{n}-t_{\beta}^{n}:\{\alpha, \beta\} \in \mathcal{L}\right) \subset R_{\Lambda}$.

If $L \subset N$ are submodules of $M$, then the isomorphism theorems may be used (see [17, Lem. 7.5]) to show that

$$
\left|N / \mathfrak{a}_{n} N\right| \leqslant\left|\frac{N / L}{\mathfrak{a}_{n}(N / L)}\right|\left|L / \mathfrak{a}_{n} L\right| .
$$

Using induction on the module chain (4.4) therefore gives

$$
\left|M / \mathfrak{a}_{n} M\right| \leqslant \prod_{i=1}^{r}\left|\frac{L_{i} / L_{i-1}}{\mathfrak{a}_{n}\left(L_{i} / L_{i-1}\right)}\right| .
$$

Set $D_{i}=R_{\Lambda} /\left(\mathfrak{q}_{i} \cap R_{\Lambda}\right), N_{i}=L_{i} / L_{i-1} \subset \mathbb{K}_{i}$ and consider this as a $D_{i}$-module in the natural way. Let $D_{i}^{\prime}$ denote the integral closure of $D_{i}$, which is a finitely generated $D_{i}$-module. For ease of notation, drop the suffix $i$, as this is fixed for the rest of the proof. Let $\left\{x_{1}, \ldots, x_{s}\right\} \subset \mathbb{K}$ be a set of generators for $D^{\prime}$ as a $D$ module and set $N^{\prime}=\sum_{j=1}^{s} x_{j} N$. Since $\mathbb{K}$ is the field of fractions of $D$, we are able to find $y \in D$, which clears the denominators of $x_{1}, \ldots, x_{s}$, giving $y D^{\prime} \subset D$, and hence $y N^{\prime} \subset N$. Furthermore, by [23, Lem. 4.3], $N^{\prime} / y N^{\prime}$ is finite and, therefore, so is $N / y N^{\prime}$. Applying (4.8) again gives

$$
\left|N / \overline{\mathfrak{a}}_{n} N\right| \leqslant\left|N / y N^{\prime}\right|\left|y N^{\prime} / \overline{\mathfrak{a}}_{n} y N^{\prime}\right| \leqslant\left|N / y N^{\prime}\right|\left|N^{\prime} / \overline{\mathfrak{a}}_{n} N^{\prime}\right|,
$$

where $\overline{\mathfrak{a}}_{n}$ is the image of $\mathfrak{a}_{n}$ in $D$. Note that the second inequality follows from the fact that $y N^{\prime} / \overline{\mathfrak{a}}_{n} y N^{\prime}$ is a homomorphic image of $N^{\prime} / \overline{\mathfrak{a}}_{n} N^{\prime}$. Again we appeal to the method of [23, Sec. 4], which can be easily adapted to show that

$$
\left|N^{\prime} / \overline{\mathfrak{a}}_{n} N^{\prime}\right|=\prod_{v \in P_{i}} \min \left\{\left|\lambda_{\alpha, i}^{n}-\lambda_{\beta, i}^{n}\right|_{v}^{-1}:\{\alpha, \beta\} \in \mathcal{L}\right\} .
$$

The main idea behind this stage of the proof is similar to that of Theorem 4.1 but is more involved because we are working with a module $N^{\prime}$ of a Dedekind domain instead of with the domain directly.

Finally, each factor in (4.9) now receives its required bound by combining (4.10) and (4.11). 
In order to provide bounds for the number of weak synchronization points, define

$$
H_{X}(\alpha, \beta)=\prod_{i=1}^{r} \prod_{v \in \mathcal{P}\left(\mathbb{K}_{i}\right) \backslash P_{i}} \max \left\{\left|\lambda_{\alpha, i}\right|_{v},\left|\lambda_{\beta, i}\right|_{v}\right\} .
$$

Theorem 4.7. Let $\Lambda$ be a finite set of commuting epimorphisms of a connected finite dimensional compact abelian group $X$ and suppose that $\widehat{\alpha}^{n}-\widehat{\beta}^{n}$ is injective for all distinct $\alpha, \beta \in \Lambda$ and all $n \in \mathbb{N}$. If every set of places $\mathcal{P}\left(\mathbb{K}_{i}\right) \backslash P_{i}, 1 \leqslant i \leqslant$ $r$, arising from a canonical filtration is finite, then for any $\alpha, \beta \in \Lambda$ there exist constants $0<A \leqslant C$ and $B \geqslant 0$ such that, for all sufficiently large $n$,

$$
\frac{A}{n^{B}} H_{X}(\alpha, \beta)^{n} \leqslant S_{n}(\alpha, \beta) \leqslant C H_{X}(\alpha, \beta)^{n} .
$$

Furthermore,

$$
r_{\Lambda}=\max _{\alpha, \beta \in \Lambda, \alpha \neq \beta}\left\{H_{X}(\alpha, \beta)\right\} .
$$

Remarks 4.8. If each of the maps in $\Lambda$ is invertible, then

$$
H_{X}(\alpha, \beta)=\prod_{i=1}^{r} H\left(\lambda_{\alpha, i}: \lambda_{\beta, i}\right)=\exp \left(h\left(\beta^{-1} \alpha\right)\right),
$$

where $H$ is the projective height and $h$ is the topological entropy. The first equality follows from invertibility of the maps in $\Lambda$ : For any $\alpha \in \Lambda$ and $1 \leqslant i \leqslant r$, invertibility of multiplication by $t_{\alpha}$ causes $\lambda_{\alpha, i}^{-1}$ to appear in $L_{i} / L_{i-1}$ under the canonical embedding (4.5) of this module into the field $\mathbb{K}_{i}$. Therefore, by the definition of $P_{i}$, for any $v \in P_{i},|\cdot|_{v}$ is bounded on $\mathbb{Z}\left[\lambda_{\alpha, i}^{ \pm 1}\right]$, which means $\left|\lambda_{\alpha, i}\right|_{v}=1$. Thus, the sets of places $\mathcal{P}\left(\mathbb{K}_{i}\right) \backslash P_{i}$ appearing in the definition of $H_{X}(\alpha, \beta)$ may be simply replaced by $\mathcal{P}\left(\mathbb{K}_{i}\right), 1 \leqslant i \leqslant r$. The second equality is given by the entropy formula of Lind and Ward 20.

Thus, the corollary agrees with Theorem 2.2, although it also applies to many non-expansive automorphisms, for example commuting quasi-hyperbolic toral automorphisms. It is prudent to note that even though the product of heights is defined if the maps are not invertible, Example 2.3 shows that this cannot be expected to give the correct growth rate for $\left|S_{n}(\Lambda)\right|$ in general.

If any set of places appearing in Theorem 4.7 is infinite, the conclusion of the theorem may or may not hold. The startling complexity of this issue may be glimpsed by taking $\Lambda=\{\alpha, \operatorname{Id}\}$ (so that $S_{n}(\Lambda)=F_{n}(\alpha)$ ) and considering the situations revealed in 8 ] and [34].

Proof of Theorem 4.7. First carry out Method 4.4 to obtain (4.6), which is valid for all $n \in \mathbb{N}$. Note that the lemma may be applied since multiplication by $t_{\alpha}^{n}-t_{\beta}^{n}$ is injective on $M$, in particular, on the submodule $R_{\Lambda} / \mathfrak{p}$ for any $\mathfrak{p} \in \operatorname{Ass}(M)$. It follows that, for $1 \leqslant i \leqslant r$ and all $n \in \mathbb{N}, \lambda_{\alpha, i}^{n} \neq \lambda_{\gamma, i}^{n}$.

To prove the required inequalities, using (4.6) it suffices to show that for each $1 \leqslant i \leqslant r$, there exist constants $0<A_{i} \leqslant C_{i}$ and $B_{i} \geqslant 0$ such that, for sufficiently large $n$,

$$
\frac{A_{i}}{n^{B_{i}}} H_{i}(\alpha, \beta)^{n} \leqslant \prod_{v \in P_{i}}\left|\lambda_{\alpha, i}^{n}-\lambda_{\beta, i}^{n}\right|_{v}^{-1} \leqslant C_{i} H_{i}(\alpha, \beta)^{n},
$$


where, setting $Q_{i}=\mathcal{P}\left(\mathbb{K}_{i}\right) \backslash P_{i}$,

$$
H_{i}(\alpha, \beta)=\prod_{v \in Q_{i}} \max \left\{\left|\lambda_{\alpha, i}\right|_{v},\left|\lambda_{\beta, i}\right|_{v}\right\} .
$$

Hence, $i$ can be fixed and subsequently dropped as a superfluous suffix.

By the product formula for global fields

$$
\prod_{v \in P}\left|\lambda_{\alpha}^{n}-\lambda_{\beta}^{n}\right|_{v}^{-1}=\prod_{v \in Q}\left|\lambda_{\alpha}^{n}-\lambda_{\beta}^{n}\right|_{v},
$$

which is a finite product. We can rewrite this as

$$
\prod_{v \in Q}\left|\lambda_{\beta}\right|_{v}^{n} \prod_{v \in Q} \underbrace{\left|\left(\frac{\lambda_{\alpha}}{\lambda_{\beta}}\right)^{n}-1\right|_{v}}_{\phi_{v}(n)} .
$$

First consider those $v \in Q$ for which $\left|\lambda_{\alpha}\right|_{v} \neq\left|\lambda_{\beta}\right|_{v}$. If $v$ is non-archimedean, the ultrametric inequality gives

$$
\phi_{v}(n)= \begin{cases}1 & \text { if }\left|\lambda_{\alpha}\right|_{v}<\left|\lambda_{\beta}\right|_{v} \\ \left|\frac{\lambda_{\alpha}}{\lambda_{\beta}}\right|_{v}^{n} & \text { if }\left|\lambda_{\alpha}\right|_{v}>\left|\lambda_{\beta}\right|_{v} .\end{cases}
$$

If $v$ is archimedean, there exists $N_{v} \in \mathbb{N}$ such that, for all $n>N_{v}$,

$$
\begin{array}{ll}
\frac{1}{2} \leqslant \phi_{v}(n) \leqslant 2 & \text { if }\left|\lambda_{\alpha}\right|_{v}<\left|\lambda_{\beta}\right|_{v}, \\
\frac{1}{2}\left|\frac{\lambda_{\alpha}}{\lambda_{\beta}}\right|_{v}^{n} \leqslant \phi_{v}(n) \leqslant 2\left|\frac{\lambda_{\alpha}}{\lambda_{\beta}}\right|_{v}^{n} & \text { if }\left|\lambda_{\alpha}\right|_{v}>\left|\lambda_{\beta}\right|_{v} .
\end{array}
$$

Now consider those $v \in Q$ for which $\left|\lambda_{\alpha}\right|_{v}=\left|\lambda_{\beta}\right|_{v}$. There exists $N_{v} \in \mathbb{N}, A_{v}>0$ and $B_{v} \geqslant 1$ such that for all $n>N_{v}$,

$$
\frac{A_{v}}{n^{B_{v}}} \leqslant \phi_{v}(n) \leqslant 2 .
$$

The first inequality follows from Baker's theorem for archimedean $v$ and a standard estimation for non-archimedean $v$ (see for example [8]).

Set $B=\sum_{v} B_{v}, A=\prod_{v} A_{v}$ and $N=\max _{v}\left\{N_{v}\right\}$. Then, for all $n>N$,

$$
\frac{A}{2^{|Q|} n^{B}} \prod_{v \in Q^{\prime}}\left|\frac{\lambda_{\alpha}}{\lambda_{\beta}}\right|_{v}^{n} \leqslant \prod_{v \in Q} \phi_{v}(n) \leqslant 2^{|Q|} \prod_{v \in Q^{\prime}}\left|\frac{\lambda_{\alpha}}{\lambda_{\beta}}\right|_{v}^{n},
$$

where $Q^{\prime}=\left\{v \in Q:\left|\lambda_{\alpha}\right|_{v}>\left|\lambda_{\beta}\right|_{v}\right\}$. Applying this to (4.14) gives (4.13), as

$$
H(\alpha, \beta)=\left(\prod_{v \in Q^{\prime}}\left|\frac{\lambda_{\alpha}}{\lambda_{\beta}}\right|_{v}\right)\left(\prod_{v \in Q}\left|\lambda_{\beta}\right|_{v}\right) .
$$

Finally, a completely analogous argument to the proof of Theorem 2.2 then gives the value for $r_{\Lambda}$.

\section{INVARIANT MEASURES AND CONNECTIONS WITH NUMBER THEORY}

In 9 , Corvaja and Zannier obtain a generalization of their bound for $\operatorname{gcd}\left(a^{n}-\right.$ $\left.1, b^{n}-1\right)$. This result has striking consequences for the rate of growth of $\left|S_{n}^{\star}(\Lambda)\right|$ when $|\Lambda| \geqslant 3$. We will use a simplified version of their full result (see [9, Eq. 1.3]). 
Theorem 5.1 (Corvaja, Zannier [9]). Let $\xi, \eta \in \overline{\mathbb{Q}}$ be multiplicatively independent. Given any $\varepsilon>0$, there are finitely many exceptions $n \in \mathbb{N}$ to the inequality

$$
\prod_{v \in \mathcal{P}(\mathbb{Q}(\xi, \eta))} \max \left\{1, \min \left\{\left|\xi^{n}-1\right|_{v}^{-1},\left|\eta^{n}-1\right|_{v}^{-1}\right\}\right\}<2^{\varepsilon n} .
$$

Combined with the results of Section 4, this gives the following.

Theorem 5.2. Let $\Lambda$ be a finite set of commuting automorphisms of a connected finite dimensional compact abelian group with $|\Lambda| \geqslant 3$. If every map of the form $\widehat{\alpha}^{m} \widehat{\beta}^{n}-\widehat{\gamma}^{m+n}$ is injective for all distinct $\alpha, \beta, \gamma \in \Lambda$ and $m, n$ not both zero, then $r_{\Lambda}^{\star}=1$.

Theorem 5.3. Let $\Lambda$ be a finite set of commuting toral automorphisms and suppose that the associated $\langle\Lambda\rangle$-action is mixing. If $|\Lambda|=2$, then $\sigma_{\Lambda}^{\star}$ is rational. If $|\Lambda| \geqslant 3$, then $\sigma_{\Lambda}^{\star}$ has meromorphic continuation to the open unit disc.

Proof of Theorem 5.2. First apply Method4.4 and note that multiplication by $t_{\alpha}^{n}-$ $t_{\beta}^{n}$ is injective for all $n \in \mathbb{N}$, by hypothesis. So, as in the proof of Theorem 4.7 for $1 \leqslant i \leqslant r$ and all $n \in \mathbb{N}, \lambda_{\alpha, i}^{n} \neq \lambda_{\gamma, i}^{n}$.

Choose three distinct maps $\alpha, \beta, \gamma \in \Lambda$. Then by (4.7),

$$
\begin{aligned}
\left|S_{n}^{\star}(\Lambda)\right| & \leqslant\left|I_{n}(\{\alpha, \gamma\},\{\beta, \gamma\})\right| \\
& \leqslant C_{i} \prod_{i=1}^{r} \prod_{v \in P_{i}} \min \left\{\left|\lambda_{\alpha, i}^{n}-\lambda_{\gamma, i}^{n}\right|_{v}^{-1},\left|\lambda_{\beta, i}^{n}-\lambda_{\gamma, i}^{n}\right|_{v}^{-1}\right\},
\end{aligned}
$$

for some $C_{i}>0$. For the moment, fix $i$ and drop this as a suffix. Invertibility of the maps in $\Lambda$ forces $\left|\lambda_{\alpha}\right|_{v}=\left|\lambda_{\beta}\right|_{v}=\left|\lambda_{\gamma}\right|_{v}=1$ for all $v \in P$ (see Remarks 4.8), and so the product over places may be written as

$$
\prod_{v \in P} \min \left\{\left|\left(\lambda_{\alpha} / \lambda_{\gamma}\right)^{n}-1\right|_{v}^{-1},\left|\left(\lambda_{\beta} / \lambda_{\gamma}\right)^{n}-1\right|_{v}^{-1}\right\}
$$

Since each factor is greater than or equal to 1 , this also equals

$$
\prod_{v \in P} \max \left\{1, \min \left\{\left|\left(\lambda_{\alpha} / \lambda_{\gamma}\right)^{n}-1\right|_{v}^{-1},\left|\left(\lambda_{\beta} / \lambda_{\gamma}\right)^{n}-1\right|_{v}^{-1}\right\}\right\} .
$$

This is clearly bounded above by the same expression with the full set of places $\mathcal{P}(\mathbb{K})$ in place of $P$. The conditions of the theorem imply that $\lambda_{\alpha}^{m} \lambda_{\beta}^{n}-\lambda_{\gamma}^{m+n}=0$ only if $m=n=0$, and consequently $\lambda_{\alpha} / \lambda_{\gamma}$ and $\lambda_{\beta} / \lambda_{\gamma}$ are multiplicatively independent. Therefore, by Theorem 5.1, given $\varepsilon>0$, there exists $N_{i} \in \mathbb{N}$ such that the product above is strictly bounded above by $2^{\varepsilon n}$ for all $n>N_{i}$. Setting $C=\prod_{i=1}^{r} C_{i}$, it follows that $\left|S_{n}^{\star}(\Lambda)\right|<C \cdot 2^{\varepsilon n}$ for all $n>\max _{1 \leqslant i \leqslant r} N_{i}$. Thus,

$$
\limsup _{n \rightarrow \infty}\left|S_{n}^{\star}(\Lambda)\right|^{1 / n} \leqslant 2^{\epsilon} .
$$

Proof of Theorem [5.3. Note first that by [30, Prop. 6.6], mixing implies ergodicity of $\widehat{\beta}^{-n} \widehat{\alpha}^{n}$ for all $n \in \mathbb{N}$ and all $\alpha, \beta \in \Lambda$. This also means $\lambda_{\beta, i}^{-n} \lambda_{\alpha, i}^{n} \neq 1$ for $1 \leqslant i \leqslant r$, permitting the use of (4.7). 
In addition to Corvaja and Zannier's bound and the results of the previous section, the essential ingredient here is Proposition 2.1(4). This shows that $\sigma_{\lambda}^{\star}$ is a sum of functions of the form

$$
\sum_{n=1}^{\infty}\left|I_{n}(\mathcal{L})\right| z^{n},
$$

where $\mathcal{L}$ is a collection of 2-element subsets of $\Lambda$ and where $I_{n}(\mathcal{L})$ is as in the proposition.

When $|\mathcal{L}|=1, I_{n}(\mathcal{L})=S_{n}(\alpha, \beta)=F_{n}\left(\beta^{-1} \alpha\right)$, for some distinct $\alpha, \beta \in \Lambda$. In this case, it is well known that $\left(\left|F_{n}\left(\beta^{-1} \alpha\right)\right|\right)$ is a recurrence sequence, so (5.1) is rational.

When $|\mathcal{L}|=2$, (4.7) shows that $\left|I_{n}(\mathcal{L})\right|$ is bounded by a constant $C>0$ times a finite product of expressions of the form

$$
\prod_{v \in P} \min \left\{\left|\lambda_{\alpha}^{n}-\lambda_{\beta}^{n}\right|_{v}^{-1},\left|\lambda_{\gamma}^{n}-\lambda_{\delta}^{n}\right|_{v}^{-1}\right\}
$$

for some $\alpha, \beta, \gamma, \delta \in \Lambda$ with $\alpha, \beta, \gamma$ distinct. This product is equal to

$$
\prod_{v \in P} \min \left\{\left|\left(\lambda_{\alpha} / \lambda_{\beta}\right)^{n}-1\right|_{v}^{-1},\left|\left(\lambda_{\gamma} / \lambda_{\delta}\right)^{n}-1\right|_{v}^{-1}\right\}
$$

as $\left|\lambda_{\beta}\right|_{v}=\left|\lambda_{\delta}\right|_{v}=1$ for all $v \in P$. The mixing assumption means that $\lambda_{\alpha} / \lambda_{\beta}$ and $\lambda_{\gamma} / \lambda_{\delta}$ are multiplicatively independent and, just as in the proof of Theorem 5.2 . Theorem 5.1 shows that for any given $\varepsilon>0$, the product is bounded above by $C \cdot 2^{\varepsilon n}$ for all sufficiently large $n$. Hence, (5.1) has radius of convergence 1 .

Finally, when $|\mathcal{L}| \geqslant 3,\left|I_{n}(\mathcal{L})\right| \leqslant\left|I_{n}\left(\mathcal{L}^{\prime}\right)\right|$ for any $\mathcal{L}^{\prime} \subset \mathcal{L}$ with $\left|\mathcal{L}^{\prime}\right|=2$, so again we conclude that (5.1) has radius of convergence 1 .

Remarks 5.4. Theorem 5.2 may or may not hold when the maps in $\Lambda$ are not invertible. To see this, consider Example 2.4 and replace the values used there with $a=2, b=6$ and $c=10$. Then $\left|S_{n}^{\star}(2,6,10)\right|$ grows exponentially fast as

$$
\left|S_{n}^{\star}(2,6,10)\right|=\operatorname{gcd}\left(10^{n}-2^{n}, 6^{n}-2^{n}\right) \geqslant 2^{n}
$$

for all $n \in \mathbb{N}$.

In general, when $|\Lambda| \geqslant 3$ and $r_{\Lambda}^{\star}=1$, there is likely to be no meromorphic continuation of $\sigma_{\Lambda}^{\star}$ beyond the unit circle because of the potentially erratic behaviour of $\left(\left|S_{n}^{\star}(\Lambda)\right|\right)$. In particular, Pólya and Carlson [7] (see also [28]) show that when a function given by a power series with integer coefficients has radius of convergence 1 , unless the coefficients are given by a recurrence sequence with polynomial growth, the unit circle is a natural boundary for the function.

To see this happen in a concrete case, again consider Example 2.4 with $a, b, c$ coprime. Then

$$
\begin{aligned}
\left|S_{n}^{\star}(a, b, c)\right| & =\operatorname{gcd}\left(c^{n}-a^{n}, b^{n}-a^{n}\right) \\
& =\prod_{p \operatorname{prime}} \min \left\{\left|c^{n}-a^{n}\right|_{p}^{-1},\left|b^{n}-a^{n}\right|_{p}^{-1}\right\} \\
& \geqslant \prod_{p \notin S} \min \left\{\left|(c / a)^{n}-1\right|_{p}^{-1},\left|(b / a)^{n}-1\right|_{p}^{-1}\right\},
\end{aligned}
$$

where $S$ is the finite set of primes for which either $|a|_{p} \neq 1,|b|_{p} \neq 1$ or $|c|_{p} \neq 1$. Note that $r_{\Lambda}^{\star}=1$, by Theorem 5.1. On the other hand, by [29] (see also [1, Prop. 10]), there is a sequence $(n(j))$ along with the number of primes such that $p-1 \mid n(j)$ is 
at least $B \log n(j) / \log \log n(j)$, for some absolute constant $B>0$. Therefore, along the sequence $n(j),(15.2)$ is at least $2^{-|S|+B \log n(j) / \log \log n(j)}$. Thus, $\left|S_{n}^{\star}(a, b, c)\right|$ grows faster than polynomially along this sequence.

In the algebraic situations considered thus far, where $X$ is a compact abelian group, we have been able to capitalize on the fact that the set of strong synchronization points is a finite $\Lambda$-invariant subgroup of $X$. As discussed in Section 2, when the maps in $\Lambda$ are invertible, this also means there is a natural associated invariant measure $\nu_{\Lambda, n}^{\star}$ given by (2.2) for each $n \in \mathbb{N}$. The problem of whether or not these measures distribute uniformly with respect to Haar measure $\nu$ (that is, whether or not the measures converge weakly to $\nu$ ) generalizes the same problem for periodic point measures. As is the case for those measures, via duality, weak convergence of $\nu_{\Lambda, n}^{\star}$ to $\nu$ is equivalent to pointwise convergence of $\widehat{\nu}_{\Lambda, n}^{\star}$ to $\widehat{\nu}$. Equivalently, for any non-zero $x \in M=\widehat{X}$, for all sufficiently large $n, \widehat{\nu}_{\Lambda, n}^{\star}(x)=0$. Furthermore, $\widehat{\nu}_{\Lambda, n}^{\star}(x)=0$ if and only if

$$
x \notin S_{n}^{\star}(\Lambda)^{\perp}=\mathfrak{a}_{n} M,
$$

where $\mathfrak{a}_{n}$ is the ideal $\left(t_{\alpha}^{n}-t_{\beta}^{n}: \alpha, \beta \in \Lambda, \alpha \neq \beta\right) \subset R_{\Lambda}$ (see [33] for details). The following theorem uses the canonical filtration introduced in Method 4.4 and generalizes well-known results for periodic point measures [18, 8]. Using the notation of Method 4.4 set

$$
\psi_{i}(n)=\prod_{v \in P_{i}}\left|\lambda_{\alpha, i}^{n}-\lambda_{\beta, i}^{n}\right|_{v}^{-1}
$$

so that (4.6) gives the factorization $\left|S_{n}(\alpha, \beta)\right|=\psi_{1}(n) \psi_{2}(n) \cdots \psi_{r}(n)$.

Theorem 5.5. Let $\alpha$ and $\beta$ be commuting automorphisms of a connected finite dimensional compact abelian group $X$ and suppose that $\widehat{\alpha}^{n}-\widehat{\beta}^{n}$ is injective for all $n \in \mathbb{N}$. If each set of places $P_{i}$ is uniformly bounded on its associated factor in a canonical filtration and $\psi_{i}(n) \rightarrow \infty, 1 \leqslant i \leqslant r$, then the measures $\nu_{\{\alpha, \beta\}, n}^{\star}$ converge weakly to Haar measure.

Proof. The notation is the same as that of Method 4.4. Let $N$ be any $R_{\{\alpha, \beta\}}$-module and define the following.

Property A. Given any non-zero $x \in N$, there exists $m(x, N) \in \mathbb{N}$ such that $x \notin\left(t_{\alpha}^{n}-t_{\beta}^{n}\right) N$ for all $n>m(x, N)$.

The result is proved by showing that Property A holds for $M=\widehat{X}$. The first step is to show that if Property A holds for $L_{i-1}$ and $L_{i} / L_{i-1}$, then it also holds for $L_{i}, 1 \leqslant i \leqslant r$. Thus, induction will show that if Property $A$ holds for each of the factors $L_{i} / L_{i-1}$, then it also holds for $M$. The second step is to show that Property A holds for each of the factors.

Step 1. Suppose Property A holds for $L_{i-1}$ and $L_{i} / L_{i-1}$. Denote the image of $y \in$ $L_{i}$ in $L_{i} / L_{i-1} \subset \mathbb{K}_{i}$ by $\bar{y}$. Let $x \in L_{i}$ be non-zero and set

$$
m\left(x, L_{i}\right)= \begin{cases}m\left(x, L_{i-1}\right) & \text { if } x \in L_{i-1}, \\ m\left(\bar{x}, L_{i} / L_{i-1}\right) & \text { if } x \notin L_{i-1}\end{cases}
$$

Let $n>m\left(x, L_{i}\right)$, write $g=t_{\alpha}^{n}-t_{\beta}^{n}$ and suppose for a contradiction that $x \in g L_{i}$. If $x \in L_{i-1}$, then $x \in g L_{i-1}$ as

$$
g L_{i} \cap L_{i-1}=g L_{i-1},
$$


which will be verified in a moment. However, this gives an immediate contradiction, as Property A holds for $L_{i-1}$. Therefore, $x \notin L_{i-1}$ and $\bar{x} \neq 0$. Since $\bar{x} \in g\left(L_{i} / L_{i-1}\right)$, again we arrive at a contradiction, as this is prohibited by Property A for $L_{i} / L_{i-1}$. Thus, we cannot have $x \in g L_{i}$, and hence Property A holds for $L_{i}$.

To verify (5.3), suppose $y \in L_{i}$. If $g y \in L_{i-1}$, then $g \bar{y}=\left(\lambda_{\alpha, i}^{n}-\lambda_{\beta, i}^{n}\right) \bar{y}=0$. Since $\lambda_{\alpha, i}^{n}-\lambda_{\beta, i}^{n} \neq 0$, this means $\bar{y}=0$, that is, $y \in L_{i-1}$.

Step 2. Let $N_{i}=L_{i} / L_{i-1}, 1 \leqslant i \leqslant r$. As usual, drop the unnecessary suffix $i$, as this is now fixed. By hypothesis, the set of places $P$ is uniformly bounded on $N$, by $A$ say. Since the places in $\mathcal{P}(\mathbb{K})$ are normalized, for any $x \in N,|x|_{v}>1$ implies $|x|_{v}>\left|\mathfrak{K}_{v}\right|$, and since there are only finitely many $v \in \mathcal{P}(\mathbb{K})$ with $\left|\mathfrak{K}_{v}\right| \leqslant A$, we must have $|N|_{v} \subset[0,1]$ for all but finitely many $v \in P$. Therefore, the function $|\cdot|_{P}=\prod_{v \in P}|\cdot|_{v}$ is bounded on $N$, by $B$ say. Suppose $x \in N$ is non-zero. If $x \in\left(\lambda_{\alpha}^{n}-\lambda_{\beta}^{n}\right) N$ for arbitrarily large $n$, then $\left(\lambda_{\alpha}^{n}-\lambda_{\beta}^{n}\right)^{-1} x \in N$, and so

$$
\psi(n)|x|_{P}=\left|\left(\lambda_{\alpha}^{n}-\lambda_{\beta}^{n}\right)^{-1} x\right|_{P} \leqslant B,
$$

for arbitrarily large $n$, contradicting $\psi(n) \rightarrow \infty$. Thus, Property A holds for $N$.

Example 7.1 of [8] shows that the condition $\psi_{i}(n) \rightarrow \infty$, for all $1 \leqslant i \leqslant r$, is a perfectly reasonable one.

The property that the sets of places are uniformly bounded is automatically satisfied if $X$ is a torus or even if $X$ simply has a finite-to-one projection onto a finite product of solenoids dual to rings of $S$-integers. Nonetheless, uniform boundedness seems hard to avoid as the following example demonstrates.

Example 5.6. Consider the group $M=\sum_{p \text { is prime }} \frac{1}{p} \mathbb{Z}\left[\frac{1}{6}\right] \subset \mathbb{Q}$, and set $\Lambda=$ $\{\times 2, \times 3\}$; these are automorphisms of the one dimensional solenoid $X=\widehat{M}$. Note that $P=\{p: p$ is prime not equal to 2 or 3$\}$, but this is not uniformly bounded on $M$, as $\frac{1}{p} \in M$ for all primes $p$. The counting formula (4.6) combined with the product formula for global fields shows that

$$
\left|S_{n}^{\star}(\Lambda)\right|=\left|S_{n}(\Lambda)\right|=\left|3^{n}-2^{n}\right|_{\infty}\left|3^{n}-2^{n}\right|_{2}\left|3^{n}-2^{n}\right|_{3}=3^{n}-2^{n},
$$

so the number of synchronization points is, once again, the familiar Lucas sequence from Example 2.3.

However, for a uniform distribution of synchronization point measures, as in the theorem above, we at least require $\left(3^{n}-2^{n}\right)^{-1} \notin M$ for all but finitely many $n \in \mathbb{N}$. This seems hard to verify, since it is not known if $3^{n}-2^{n}$ is prime infinitely often. If this were the case, then clearly the measures would not uniformly distribute. More generally, in order to show that the measures do not uniformly distribute, we need $x \in M$ such that $\left(3^{n}-2^{n}\right)^{-1} x \in M$ (so, $p^{2} \nmid 3^{n}-2^{n}$ for all primes $p \nmid 6 x$ ) for infinitely many $n \in \mathbb{N}$. This also appears to be a difficult problem. See A001047 and A057468 in the On-line Encyclopedia of Integer Sequences 31 for further details on the specific Lucas sequence $\left(3^{n}-2^{n}\right)$ and [13, Ch. 6] for problems concerning primes occurring in more general recurrence sequences of this type.

In light of the example above, it would be interesting to know if there are any one dimensional solenoids upon which the number of synchronization points for $\times 2, \times 3$ grows exponentially fast, yet the synchronization point measures do not converge weakly to Haar measure. 
For $|\Lambda| \geqslant 3$, weak convergence of the measures $\nu_{\Lambda, n}^{\star}$ to Haar measure obviously depends heavily on the behaviour of expressions such as (4.1). In particular, if the following conjecture of Ailon and Rudnick [2] holds, it provides examples with $|\Lambda|=3$ that contrast dramatically with Theorem [5.5. tentatively suggesting the "typical" picture in this case.

Conjecture 5.7 (Ailon, Rudnick [2]). If $a, b$ are multiplicatively independent nonzero integers with $\operatorname{gcd}(a-1, b-1)=1$, then there are infinitely many $n \in \mathbb{N}$ such that $\operatorname{gcd}\left(a^{n}-1, b^{n}-1\right)=1$.

Corollary 5.8. If Conjecture [5.7 holds, then for the set of automorphisms $\Lambda=$ $\{\times a, \times b, \times 1\}$ of the one dimensional solenoid dual to $\mathbb{Z}\left[\frac{1}{a b}\right]$, the synchronization point measure $\nu_{\Lambda, n}^{\star}$ is the Dirac mass at zero for infinitely many $n \in \mathbb{N}$.

\section{REFERENCES}

1. L. M. Adleman, C. Pomerance, and R. S. Rumely. On distinguishing prime numbers from composite numbers. Ann. of Math. (2), 117(1):173-206, 1983. MR683806 (84e:10008)

2. N. Ailon and Z. Rudnick. Torsion points on curves and common divisors of $a^{k}-1$ and $b^{k}-1$. Acta Arith., 113(1):31-38, 2004. MR2046966 (2004m:11045)

3. R. Bowen. Periodic points and measures for Axiom A diffeomorphisms. Trans. Amer. Math. Soc., 154:377-397, 1971. MR0282372(43:8084)

4. R. Bowen. Some systems with unique equilibrium states. Math. Systems Theory, 8(3):193-202, 1974/75. MR0399413(53:3257)

5. M. Boyle and D. Lind. Expansive subdynamics. Trans. Amer. Math. Soc., 349(1):55-102, 1997. MR1355295 (97d:58115)

6. Y. Bugeaud, P. Corvaja, and U. Zannier. An upper bound for the G.C.D. of $a^{n}-1$ and $b^{n}-1$. Math. Z., 243(1):79-84, 2003. MR1953049(2004a:11064)

7. F. Carlson. Über ganzwertige Funktionen. Math. Z., 11(1-2):1-23, 1921. MR1544479

8. V. Chothi, G. Everest, and T. Ward. S-integer dynamical systems: Periodic points. J. Reine Angew. Math., 489:99-132, 1997. MR1461206 (99b:11089)

9. P. Corvaja and U. Zannier. A lower bound for the height of a rational function at $S$-unit points. Monatsh. Math., 144(3):203-224, 2005. MR2130274 (2005k:11140)

10. M. Einsiedler and D. Lind. Algebraic $\mathbb{Z}^{d}$-actions of entropy rank one. Trans. Amer. Math. Soc., 356(5):1799-1831 (electronic), 2004. MR2031042 (2005a:37009)

11. M. Einsiedler, D. Lind, R. Miles, and T. Ward. Expansive subdynamics for algebraic $\mathbb{Z}^{d}$ actions. Ergodic Theory Dynam. Systems, 21(6):1695-1729, 2001. MR1869066 (2004c:37033)

12. G. Everest, V. Stangoe, and T. Ward. Orbit counting with an isometric direction. In Algebraic and topological dynamics, volume 385 of Contemp. Math., pages 293-302. Amer. Math. Soc., Providence, RI, 2005. MR2180241(2006k:37046)

13. G. Everest, A. van der Poorten, I. Shparlinski, and T. Ward. Recurrence sequences, volume 104 of Mathematical Surveys and Monographs. American Mathematical Society, Providence, RI, 2003. MR1990179(2004c:11015)

14. S. Friedland. Entropy of graphs, semigroups and groups. In Ergodic theory of $\mathbf{Z}^{d}$ actions (Warwick, 1993-1994), volume 228 of London Math. Soc. Lecture Note Ser., pages 319-343. Cambridge Univ. Press, Cambridge, 1996. MR.1411226 (97f:58080)

15. W. Geller and M. Pollicott. An entropy for $\mathbb{Z}^{2}$-actions with finite entropy generators. Fund. Math., 157(2-3):209-220, 1998. Dedicated to the memory of Wiesław Szlenk. MR,1636888 (99h:58105)

16. Y. Kim, J. Lee, and K. Park. A zeta function for flip systems. Pacific J. Math., 209(2):289-301, 2003. MR.1978372(2004f:37011)

17. D. Lind, K. Schmidt, and T. Ward. Mahler measure and entropy for commuting automorphisms of compact groups. Invent. Math., 101(3):593-629, 1990. MR1062797 (92j:22013)

18. D. A. Lind. Dynamical properties of quasihyperbolic toral automorphisms. Ergodic Theory Dynamical Systems, 2(1):49-68, 1982. MR684244 (84g:28017) 
19. D. A. Lind. A zeta function for $\mathbf{Z}^{d}$-actions. In Ergodic theory of $\mathbf{Z}^{d}$ actions (Warwick, 19931994), volume 228 of London Math. Soc. Lecture Note Ser., pages 433-450. Cambridge Univ. Press, Cambridge, 1996. MR1411232 (97e:58185)

20. D. A. Lind and T. Ward. Automorphisms of solenoids and p-adic entropy. Ergodic Theory Dynam. Systems, 8(3):411-419, 1988. MR961739 (90a:28031)

21. H. Matsumura. Commutative ring theory, volume 8 of Cambridge Studies in Advanced Mathematics. Cambridge University Press, Cambridge, second edition, 1989. MR.1011461 (90i:13001)

22. R. Miles. Zeta functions for elements of entropy rank-one actions. Ergodic Theory Dynam. Systems, 27(2):567-582, 2007. MR2308145(2008h:37008)

23. R. Miles. Periodic points of endomorphisms on solenoids and related groups. Bull. Lond. Math. Soc., 40(4):696-704, 2008. MR2441142 (2009e:37015)

24. R. Miles and T. Ward. Periodic point data detects subdynamics in entropy rank one. Ergodic Theory Dynam. Systems, 26(6):1913-1930, 2006. MR2279271(2010h:37016)

25. R. Miles and T. Ward. Uniform periodic point growth in entropy rank one. Proc. Amer. Math. Soc., 136(1):359-365 (electronic), 2008. MR2350424(2009g:37005)

26. R. Miles and T. Ward. Orbit-counting for nilpotent group shifts. Proc. Amer. Math. Soc., 137(4):1499-1507, 2009. MR2465676(2010b:37011)

27. J. Milnor. On the entropy geometry of cellular automata. Complex Systems, 2(3):357-385, 1988. MR955558 (90c:54026)

28. R. Morikawa. On power series with integer coefficients. J. Fac. Sci. Univ. Tokyo Sect. IA Math., 22(2):229-235, 1975. MR0382611(52:3493)

29. K. Prachar. Über die Anzahl der Teiler einer natürlichen Zahl, welche die Form $p-1$ haben. Monatsh. Math., 59:91-97, 1955. MR0068569(16:904h)

30. K. Schmidt. Dynamical systems of algebraic origin, volume 128 of Progress in Mathematics. Birkhäuser Verlag, Basel, 1995. MR1345152 (97c:28041)

31. N. J. A. Sloane. The on-line encyclopedia of integer sequences. Notices Amer. Math. Soc., 50(8):912-915, 2003. MR1992789 (2004f:11151)

32. S. Smale. Differentiable dynamical systems. Bull. Amer. Math. Soc., 73:747-817, 1967. MR0228014 (37:3598)

33. T. Ward. Periodic points for expansive actions of $\mathbf{Z}^{d}$ on compact abelian groups. Bull. London Math. Soc., 24(4):317-324, 1992. MR.1165372(93h:22010)

34. T. Ward. Almost all $S$-integer dynamical systems have many periodic points. Ergodic Theory Dynam. Systems, 18(2):471-486, 1998. MR1619569(99k:58152)

School of Mathematics, University of East Anglia, Norwich, NR4 7TJ, United KingDOM

E-mail address: r.miles@uea.ac.uk 